\title{
Perosomus elumbis asociado a hidrocefalia, anomalías urogenitales y ano-rectales en un feto mular
}

\section{Perosomus elumbis associated to hydrocephalus, urogenital and anorectal anomalies in a mule fetus}

\author{
Teresa Oviedo S, ${ }^{1 *}$ M.Sc, Marco González T, ${ }^{1}$ M.Sc, Misael Oviedo P, ${ }^{2}$ Esp. \\ ${ }^{1}$ Universidad de Córdoba, Facultad de Medicina Veterinaria y Zootecnia, Departamento \\ de Ciencias Pecuarias. Montería, Colombia. ${ }^{2}$ Ejercicio profesional. * Correspondencia: \\ tere_oviedo@yahoo.com
}

Recibido: Octubre 20 de 2009; Aceptado: Mayo 19 de 2010.

\section{RESUMEN}

El presente caso describe el primer reporte de literatura de una rara anomalía congénita denominada Perosomus elumbis, asociada a severa hidrocefalia en un feto mular. Perosomus elumbis es una alteración de etiología desconocida caracterizada por la agenesia parcial o completa de las vértebras lumbares, sacras y coccígeas, en estos casos, la médula espinal termina en un canal vertebral ciego. Frecuentemente está asociada con artrogriposis de los miembros posteriores y malformaciones de la musculatura.

Palabras clave: Perosomus elumbis, hidrocefalia, anomalía fetal, Colombia.

\section{ABSTRACT}

This case describes the first report in the literature of a rare congenital anomaly called Perosomus elumbis associated with severe hydrocephalus in a mule fetus. Perosomus elumbis is a disorder of unknown etiology characterized by partial or complete agenesis of the lumbar, sacral and coccyges vertebrae, in these cases, the spinal cord ends in a blind vertebral canal. Perosomus elumbis is often associated to arthrogryposis of the hind limbs and malformations of the musculature.

Key words: Perosomus elumbis, hydrocephalus, fetal anomaly, Colombia. 


\section{NTRODUCCI ÓN}

El concepto de Perosomus elumbis hace referencia a un conjunto raro de malformaciones congénitas que incluyen agenesia parcial o completa de las vértebras lumbares, sacras y coccígeas, donde la médula espinal termina en un canal vertebral ciego. Con frecuencia esta alteración está asociada con artrogriposis de los miembros posteriores y malformaciones de la musculatura. La alteración ha sido reportada principalmente en las especies bovina, ovina, porcina y canina (1-6).

Existen descripciones que indican la participación de otros sistemas del cuerpo en casos de Perosomus elumbis, como anomalías urogenitales y ano-rectales (1).

El primer reporte de Perosomus elumbis en la teratología veterinaria fue realizado en el año 1832 por el patólogo y anatomista Ernst Gurlt (7), quien reportó la malformación en un ternero, desde entonces, esta rara malformación constituye un desafío para determinar aspectos relacionados con su patogénesis y etiología, en gran parte desconocidos.

Por otra parte, la hidrocefalia es una patología neonatal generalmente congénita de común presentación en los animales, que en términos generales se relaciona con todo evento que bloquee el flujo del líquido cefalorraquídeo ( LCR), especialmente a través del acueducto mesencefálico o las aberturas laterales del cuarto ventrículo (8). Lo anterior, se manifiesta con un crecimiento excesivo del cráneo y un aspecto abombado del mismo. La hidrocefalia congénita tiene diversas causas, muchas de las cuales no están completamente esclarecidas. Cabe anotar, que las malformaciones u obstrucciones de las vías que transportan el LCR, procesos inflamatorios, masas tumorales, infecciones virales, toxinas, e incluso trastornos nutricionales pueden participar en el origen de esta alteración (8-10).

El presente caso tiene el propósito de describir el primer reporte en Colombia y quizás a nivel mundial de Perosomus elumbis, asociado a otras anomalías particularmente, hidrocefalia y malformación urogenital y anorectal en un feto mular abortado.

\section{PRESENTACI ÓN DEL CASO}

Antecedentes. Se solicitó ayuda médica veterinaria para resolución de un caso de aborto distócico en una yegua de raza criolla, de 6 años de edad, peso aproximado de $350 \mathrm{~kg}$ y 40 parto, que fue servida por monta natural con un asno. Durante la gestación se mantuvo en un potrero con pasto Colosuana (Botriochloa pertusa), agua ad libitum y no se suministró ningún tipo de suplementación proteica o mineral. Fue desparasitada con un producto de uso oral que contenía pamoato de pirantel y praziquantel y la única vacunación reportada fue contra encefalitis equina.

Examen clínico e inspección. La yegua se encontró agitada, con sudoración, inquietud y signos de dolor que se manifestaron con cambios de posición de la cabeza y miembros posteriores. Se observó la exteriorización parcial hasta la región torácica de un feto en posición caudal (Figura 1). Se tomaron las constantes fisiológicas reportándose frecuencia respiratoria: 23 respiraciones/ minuto, frecuencia cardíaca: $55 \mathrm{l} /$ minuto y pulso fuerte. Se realizó palpación rectal, y se determinó un aumento anormal del tamaño cefálico fetal.

El procedimiento médico-farmacológico consistió en canalización de la vena yugular con el fin de administrar solución fisiológica al

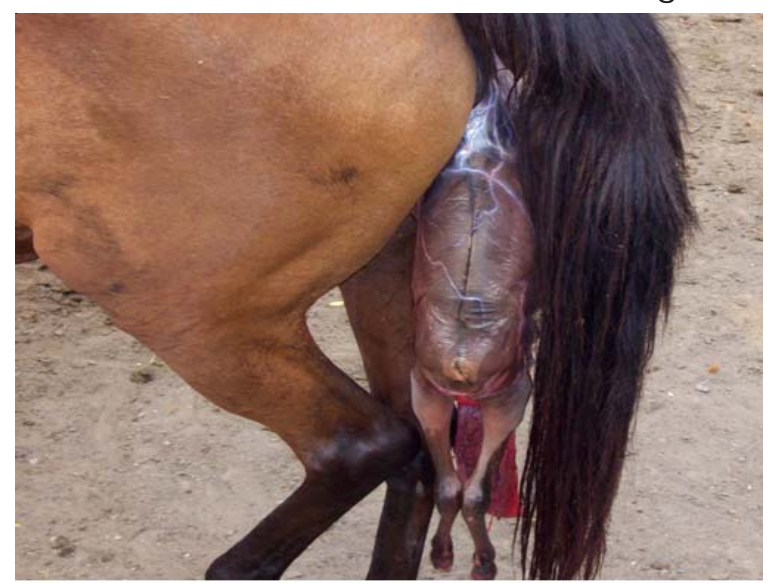

Figura 1. Parto distócico en yegua por malformación fetal. 
0.9\% y simultáneamente por la misma vía se administró flunixin meglumina a dosis de 1.1 $\mathrm{mg} / \mathrm{kg}$. Para el procedimiento obstétrico se aplicó 150 mg de lidocaína vía epidural baja. Se realizó episiotomía y luego de 20 minutos de procedimientos obstétricos se extrajo el feto. Posteriormente se evidenció la normalización de las constantes fisiológicas de la yegua y se finalizó con la sutura de la vulva con puntos en U.

El feto fue trasladado a la Facultad de Medicina Veterinaria y Zootecnia de la Universidad de Córdoba para su estudio correspondiente.

Examen externo. Se recibe para estudio un feto mular de sexo macho, el cual teniendo en cuenta las características externas descritas por Ginther (11) para determinar la edad de fetos equinos, sugiere una edad aproximada de 270 días de gestación. A la inspección externa de cabeza y rostro del animal no se apreciaron los rasgos morfológicos típicos de los équidos, se observó una bóveda craneana con gran aumento de tamaño y evidencia de contenido líquido. Debido al incremento en el tamaño del cráneo, se deformaron completamente las otras estructuras de la cabeza, es así, como no hubo evidencias del globo ocular, no obstante, se apreciaron en la piel estructuras correspondientes a la fisura palpebral (Figuras 2 y 3 ).

Estructuras correspondientes al maxilar y la mandíbula se observaron deformadas y no hubo evidencia de vías respiratorias. Las orejas estaban desplazadas completamente



Figura 2. Feto mular abortado con incremento en el tamaño cefálico y agenesia de la cauda.

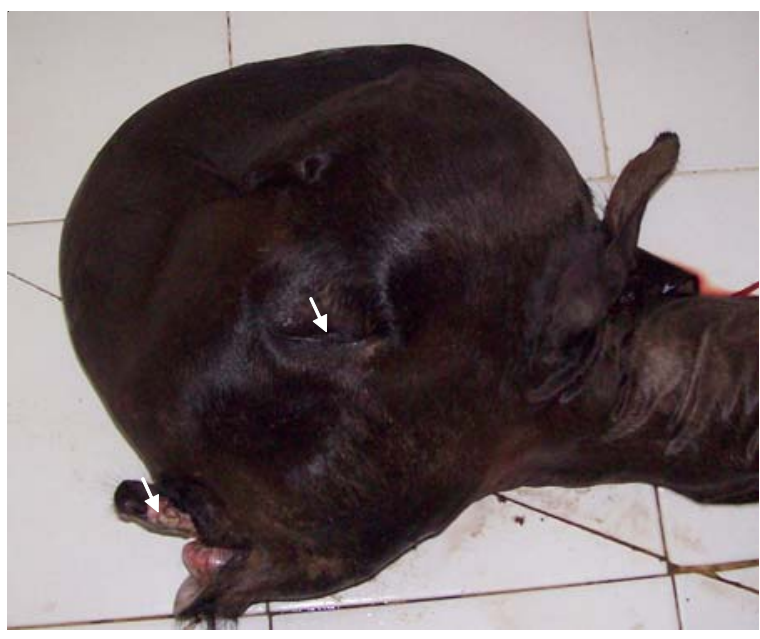

Figura 3. Rostro de feto mular abortado donde se aprecia fisura palpebral y estructuras de la boca (flechas).

hacia la parte caudal de la cabeza. Otras alteraciones observadas, consistieron en agenesia de la cola, una depresión en la línea media de la región sacra de la columna vertebral y estenosis del ano.

Examen radiográfico. El examen radiográfico del feto permitió la observación de una bóveda craneal de densidad homogénea donde se apreció claramente la abertura extrema de las fontanelas, se observó un incremento de tamaño de la bóveda craneana, especialmente hacia la parte anterior de la cabeza haciendo difícil identificar la anatomía radiográfica esencial del cráneo. El maxilar y la rama horizontal de la mandíbula se diferenciaron básicamente por la presencia de los alvéolos dentarios, no obstante, no se observó la morfología típica de estas estructuras (Figura 4).

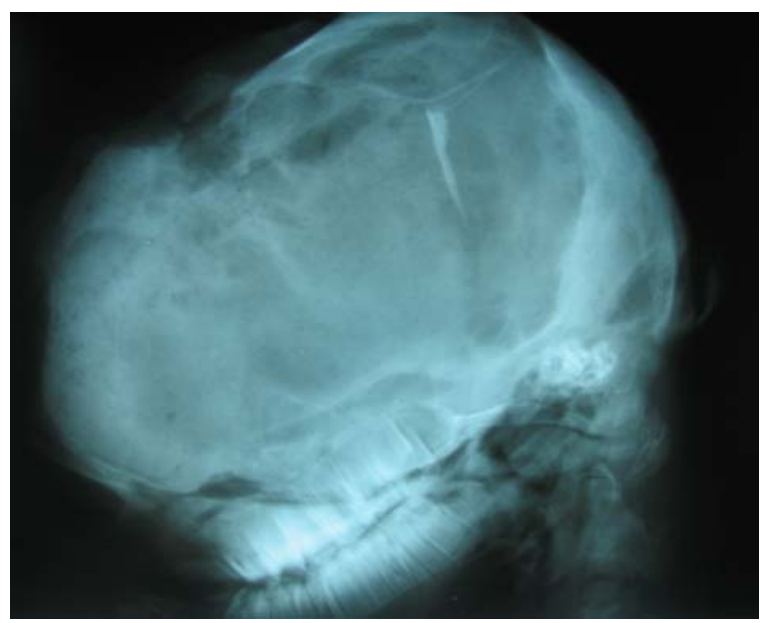

Figura 4. Radiografía de cabeza donde se aprecian alveolos dentarios y deformación extrema del cráneo. 
Las vértebras cervicales, torácicas y lumbares se observaron normales, sin embargo, no hubo signos radiológicos de la zona sacra y coccígea. La región pélvica y los miembros posteriores se observaron unidos al cuerpo por tejidos blandos (Figura 5).



Figura 5. Radiografía lateral de feto mular donde se aprecia la ausencia de vértebras sacras y coccígeas (flecha).

Hallazgos de necropsia. A la necropsia se constató la presencia de líquido en la cavidad craneal, las paredes de la misma abarcaban todo el diámetro de la cabeza con ausencia total de los senos frontal y maxilar, estructuras oculares y nasales. Un hecho que llamó mucho la atención fue la atrofia y aplastamiento de la masa encefálica, la cual tenía aspecto de torta con aproximadamente dos centímetros de espesor (Figura 6). Otros hallazgos

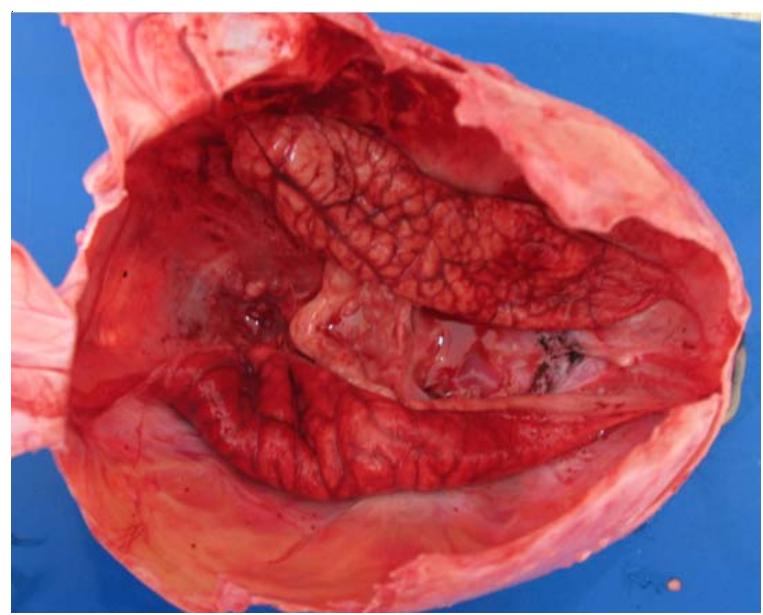

Figura 6. Aspecto del cráneo a la necropsia donde se observa aplastamiento de la masa encefálica. importantes a la necropsia fueron la estenosis anorectal, la agenesia unilateral de riñón (Figura 7) y la terminación de las vértebras en la región lumbar correspondiente a L6, hecho que permite diagnosticar el caso en estudio como Perosomus elumbis. Los demás órganos de las cavidades torácica y abdominal se encontraron normales macroscópicamente.

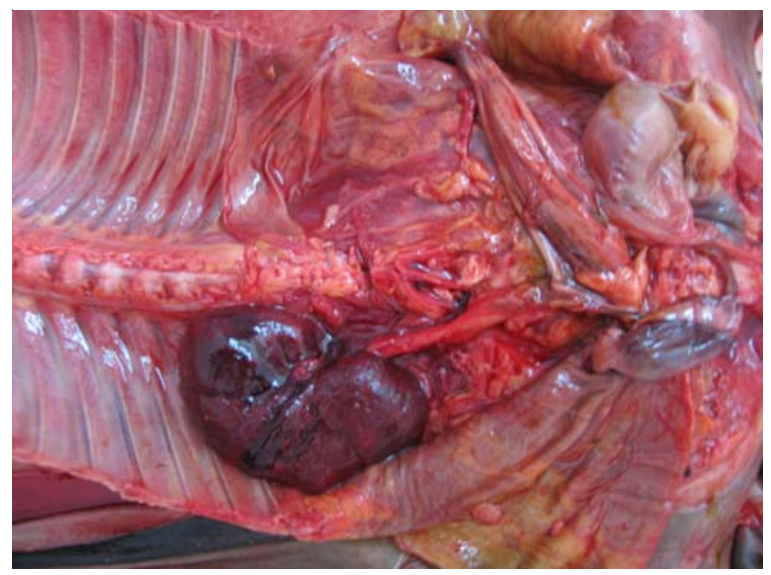

Figura 7. Agenesia unilateral de riñón en feto mular equino asociada a Perosomus elumbis.

\section{DISCUSIÓN}

Perosomus elumbis es una alteración que ha sido reportada con mayor frecuencia en las especies bovina y ovina $(1-3,12-15)$ y menos en porcinos (5). A la fecha, no se conocen reportes en híbridos como el mulo. Los defectos congénitos pueden ser el resultado de perturbaciones del desarrollo embrionario y fetal. Al nacimiento, estos defectos pueden manifestarse en un tipo particular de células, órgano o sistema o pueden involucrar varios sistemas del organismo. Al respecto, un estudio de Greene et al (16), en 1.275 terneros reporta la alteración congénita del sistema óseo en el $37 \%$ de los animales estudiados, mientras que los defectos congénitos multiorgánicos relacionados con Perosomus elumbis como el observado en el presente caso, corresponden a menos del $1 \%$.

Un hecho interesante del presente reporte es la asociación de hidrocefalia con Perosomus elumbis, lo que sugiere una alteración en el flujo de LCR; al respecto, no fue posible determinar la situación 
específica que conllevó a la presentación de hidrocefalia, puesto que el contenido de líquido intracraneal no estaba dentro de los ventrículos sino fuera de ellos lo cual ocasionó una severa presión y deformación del encéfalo y demás estructuras de la cabeza, dificultando su estudio. Aunque no se ha encontrado literatura que asocie la hidrocefalia con casos de Perosomus elumbis, Testoni et al (17), reportaron en un ternero la presencia de un Foramen magnum amplio con exposición del cerebelo y parte posterior de los hemisferios cerebrales.

Rieck et al (18) hacen referencia por primera vez en 1973 al denominado "Síndrome caudorectourogenital" asociado a defectos neuroesqueléticos como el Perosomus elumbis, posteriormente, Hiraga y Abe (19) y J ones (1) reportaron esta misma malformación asociada con anomalías urogenitales y anorectales como agenesia unilateral de riñón y atresia anal, lo cual coincide con el presente estudio, diferenciándose que en este caso no presentó atresia anal, sino estenosis.

Los mecanismos patogénicos relacionados con la formación del Perosomus elumbis no se conocen plenamente. Fue sugerido que la migración inadecuada del tubo neural, el fallo en el cierre del surco neural y del neuroporo podrían estar relacionados con la anomalía que resulta en la agenesia parcial de la columna vertebral $(1,20)$. En Australia, esta anomalía se relaciona con el consumo de la planta Veratrum californicum entre el los días 16 y 17 de gestación (3).

Los hallazgos radiológicos y de necropsia permitieron diagnosticar el presente caso como Perosomus elumbis asociado con hidrocefalia, alteraciones urogenitales y anorectales, y se considera el primer reporte de esta malformación en feto mular.

\section{REFERENCI AS}

1. Jones CJ. Perosomus elumbis (vertebral agenesis and arthrogriposis) in stillborn Holstein calf. Vet Pathol 1999; 24(1):6470.

2. Dennis SM. Perinatal lamb mortality in Western Australia: 7 Congenital defect. Australian VetJ 1975a; 51(2):80-82.

3. Dennis SM. Perosomus elumbis in sheep. Australian Vet J 1975b; 51(3): 135-136.

4. Dennis SM. Congenital defects of the nervous system of lambs. Australian Vet J 1975c; 51(9): 385-388.

5. Avedillo LJ, Camon J. Perosomus elumbis in a pig. The Veterinary Record 2007 160(4): 127-129.

6. Araújo $B M$, Kemper $B$, Figueiredo $M L$, Chioratto R, Tudury EA. Perosomus elumbus em cão Beagle. Ciênc vet tróp 2008; 11(1):36- 39.
7. Gurlt EF, Lehrbuch der pathogischen Anatomie der Haus-Säugethiere, 2. Teil, Atlas. Berlin, Germany; Ed Reimer: 1832.

8. Summers BA, Cummings JF, de Lahunta A. Veterinary Neuropathology, St. Louis, MO; Mosby-Year Book: 1995.

9. Muñana KR. Capítulo alteraciones del cerebro. En: Morgan RV, Bright RM, Swartout MS. Clínica de pequeños animales 4a ed. ELSEVIER: 2003.

10. Baumgartner WK, Krakowka S, Koestner A, Evermann J. Acute encephalitis and hydrocephalus in dogs caused by canine parainfluenza virus. Vet Pathol 1982; 19: 79-92.

11. Ginther OJ . Reproductive Biology of the mare basic and applied aspects $2^{a}$ Ed. Wisconsin USA: Ed. Equi services; 1992. 
12. Greene HJ, Leipold HW, Dennis SM. Perosomus elumbis in Hereford calves. Vet Med Small Anim Clin 1973a; 68: 167168.

13. Castro MB, Szabo MPJ, Hokamura HK, Romano MA. Perosomus elumbis in a Holstein calf in Brazil. Vet Rec 2003; 152(24): 753-753.

14. Dennis SM. Perinatal lamb mortality in western Australia. Australian VetJ 1974; 50:443-449.

15. Botelho de Castro M, Juan MS, Coelho $M A$, J unqueira BJ, Perosomus elumbis em um cordeiro no Brasil. Ciência Rural 2008; 38(1):262-265.

16. Greene HJ , Leipold HW, Huston K, Noordsy $\mathrm{JL}$, Dennis SM. Congenital defects in cattle. Ir Vet J 1973b; 27:37-45.

17. Testoni S, Mazzariol A, Gentile. Perosomus elumbis in quattro vitelli. 13응 Congreso Internazionale della Congreso Internazionale della Federazione Mediterranea Sanitá e Produzione Ruminati (FE.ME.S.P.RUM). Facoltà di Medicina Veterinaria, Valenzano. Bari 2005.
18. Rieck GW, Herzog A y Rau W. Untersuchungen zur terato-logischen Populationsstatistik und zur Atiologie der embryonalen Entwicklungsstomngen beim Rind. II. Mifibildungs syndrome. Giessener Beitrage fur Erbpathologie und Zuchthygiene. 1973; 5: 1-70.

19. Hiraga T, Abe M. A case of Perosomus elumbis in a calf. J Jpn Vet Med Assoc 1983; 36:277-279.

20. Hiraga T, Abe M. Anatomical observation of six calves affected with segmental aplasia of the spinal cord. Anat Record 1987; 219(4): 402-408. 